\title{
Von der Lehr- zur Lernorientierung - auf dem Weg zu einer rechtswissenschaftlichen Fachdidaktik
}

\author{
Von Rechtsanwältin Judith Brockmann, Wiss. Mit. Jan-Hendrik Dietrich und Wiss. Mit. Arne Pilniok, Hamburg*
}

Die Verbesserung der Hochschullehre gerät an den juristischen Fakultäten in Deutschland zunehmend in den Blick. Der Beitrag widmet sich Grundsatzfragen der Wissensvermittlung und Wissensaneignung im rechtswissenschaftlichen Studium.

»Wo bleibt die Rechtsdidaktik?« hat jüngst Klaus F. Röhl gefragt ${ }^{1}$. In den anglo-amerikanischen Hochschulen ist die Antwort eindeutig. Dort ist selbstverständlich von legal education die Rede; ihr sind Professuren, Tagungen, Zeitschriften und Bücher gewidmet. In Deutschland muss man wohl antworten: das ist ein Schatz, der noch zu heben ist. Im Folgenden wird für die Entwicklung einer rechtswissenschaftlichen Fachdidaktik plädiert. Zunächst soll dazu ein Blick auf die strukturellen Faktoren geworfen werden, die das Entstehen einer rechtswissenschaftlichen Fachdidaktik bisher behindert haben und künftig befördern könnten (I.). AnschlieBend sind inhaltliche Elemente einer rechtswissenschaftlichen Fachdidaktik zu skizzieren, die als erste Bausteine entwickelt werden könnten (II.). Neben einer inhaltlichen Vertiefung ist allerdings auch eine personelle, institutionelle und kommunikative Infrastruktur für rechtswissenschaftliche Fachdidaktik notwendig (III.).

\section{Rechtswissenschaftliche Fachdidaktik zwischen Hoch- schuldidaktik, Systemdebatte und Universitätsreformen}

\section{Hochschuldidaktik ohne fachspezifischen Zugang}

In den Nachwehen der Studentenbewegung wurde auch die Lehre ein Thema, wenngleich unter anderen Vorzeichen als heute. Sichtbarer Ausdruck dessen war die Institutionalisierung von Hochschuldidaktikzentren, die als zentrale Einrichtungen den Rahmen für eine Verbesserung der Lehre darstellen sollten. An zahlreichen deutschen Universitäten wurden entsprechende Zentren eingerichtet - häufig mit dem Zusatz »interdisziplinär«. Eine Erfolgsgeschichte sind diese Zentren für Hochschuldidaktik freilich nicht geworden. Dafür sind zwei zentrale Probleme auszumachen, die der Wissenschaftsrat jüngst noch einmal herausgestellt hat $^{2}$. Zum einen konstatiert er eine Verwissenschaftlichung der Didaktikzentren durch eine starke Orientierung an wissenschaftlicher Reputation, die hauptsächlich für Forschungsleistungen in der Erziehungswissenschaft und Psychologie erlangt werden $\mathrm{kann}^{3}$. In der Konsequenz hat, so der Wissenschaftsrat zutreffend, der Dienstleistungsauftrag der Didaktikzentren gelitten. Mit dieser engen Bindung an die Forschung hängt das zweite Problem zusammen. Es ist den Didaktikzentren nicht gelungen, ihre Tätigkeiten hinreichend disziplinenspezifisch auszudifferenzieren. Neben der Hinwendung zu erziehungswissenschaftlich-psychologisch orientierten Fachgemeinschaften liegt ein Grund dafür in den rasch wieder abgesunkenen finanziellen und personellen Ressourcen für die Didaktikzentren. So blieb etwa der Hamburger Plan, Professuren einzurichten, die jeweils mit einem halben Deputat an einem der Fachbereiche und am Didaktikzentrum tätig sind und so die Einbettung in die jeweiligen Fachbereiche und ihre Fachgemeinschaften sichern sollten, unverwirklicht. Daraus resultieren bis heute erhebliche Übersetzungsschwierigkeiten, wenn es um den Transfer hochschuldidaktischer Erkenntnisse zur Lehr- und Lernpsychologie in fachspezifische Unterrichtssituationen geht. Die hochschuldidaktischen Konzepte und Angebote richten sich an Leitbildern und Formen des akademischen Unterrichts in nur wenigen Studiengängen aus. Dies führt zu Abwehrhaltungen, weil fachspezifische Traditionen und Rah- menbedingungen der Lehre nicht hinreichend berücksichtigt werden ${ }^{4}$. Gleichwohl sollten die hochschuldidaktischen Erkenntnisse zu Selbstreflexionen in der juristischen Lehre Anlass geben. Nicht alles, was seit Jahrzehnten in der rechtswissenschaftlichen Ausbildung üblich ist, dürfte auch bewahrenswert sein.

\section{Die Debatte um die Juristenausbildung: Konzentration auf die Systemfrage}

Die Ausbildung der Juristen ist ein beständiges Thema der Fachgemeinschaft. Im Vordergrund der Debatte steht allerdings schon seit Jahrzehnten die Systemfrage der juristischen Ausbildung. Eine Auseinandersetzung über Inhalte ist damit zwar untrennbar verknüpft, so etwa die Integration von Rechts- und Sozialwissenschaften, die $\mathrm{zu}$ den programmatischen Neuerungen der einstufigen Juristenausbildung zählte. Selten ging und geht es in diesen Debatten aber um die Vermittlung der Inhalte. Dies gilt - wenn auch mit Nuancen - für die Diskussion um die einstufige Juristenausbildung in den 1970ern wie auch für die momentane Debatte um die Einführung einer BachelorMaster-Studienstruktur in der Rechtswissenschaft. Lediglich vereinzelt finden sich (rechts-)didaktische Überlegungen und Anregungen in der Literatur.

Hoffmann-Riem etwa verweist im Rückblick auf $»$ neue[n] didaktische Formen « als ein Element der Reformbemühungen der einstufigen Juristenausbildung in Hamburg ${ }^{5}$. Dies drängte sich auch deshalb auf, weil die einstufige Juristenausbildung jedenfalls in ihren Anfängen über eine wesentlich bessere Betreuungsrelation verfügte als die herkömmlichen Studiengänge. Eine Abkehr von der Großvorlesung lag deshalb nahe, war aber eben auch personell möglich. Betrachtet man den im Rahmen der einstufigen Juristenausbildung praktizierten Rechtsunterricht näher, finden sich eine Reihe von didaktischen Überlegungen, die in Vergessenheit geraten sind. Beispielsweise enthalten die Materialien zur einstufigen Ausbildung in Bremen Überlegungen zum problemorientierten Projektunterricht ${ }^{6}$, die - befreit man sie von der dekadentypischen Sprache und ideologischem Ballast - auch heute noch sinnvoll sind, um mit dem Konzept des exemplarischen Lernens, etwa in den neu gestalteten Wahlschwerpunkten ${ }^{7}$, ernst zu machen.

Auch die aktuelle Debatte über die Integration der deutschen Juristenausbildung in den Bologna-Prozess widmet sich nahezu

* Der Beitrag ist während der gemeinsamen Tätigkeit der Verf. als wissenschaftliche Mitarbeiter an der Fakultät für Rechtswissenschaft der Universität Hamburg entstanden. Inzwischen ist Judith Brockmann Rechtsanwältin und Lehrbeauftragte an der Universität Hamburg, der Leibniz Universität Hannover und der Ludwigs-Maximilians-Universität München.

1 RöHL Wo bleibt die Rechtsdidaktik?, http://rsozblog.de/?cat=90 (Stand 14. März 2009).

2 Wissenschaftsrat Empfehlungen zur Qualitätsverbesserung von Lehre und Studium, WR-Drs 8639-08, 45.

3 Wissenschaftsrat (Fn. 2) 69.

4 Das gilt für die Rechtswissenschaft, dürfte aber ebenso etwa für die Naturwissenschaften zutreffen.

5 Hoffmann-Riem JZ 2007, 645 (646); vgl. auch die knappe Skizze bei SonNen in: Pressestelle der Universität Hamburg (Hrsg.) Das Hamburger Modell der einstufigen Juristenausbildung in der Bewährung 1981, $55 \mathrm{ff}$.

6 Vgl. etwa Däubler in: Rinken et al., Der neue Jurist: Materialien zur reformierten Juristenausbildung in Bremen 1973, $158 \mathrm{ff}$.

7 Siehe hierzu die Übersicht bei Rolfs/Rossi-Wilberg JuS 2007, 297 (300 ff.) 
ausschließlich der Systemfrage. So thematisieren die in der Literatur reichhaltig vorfindlichen Beiträge etwa, ob für Absolventen eines juristischen Bachelorstudiums überhaupt ein Bedarf auf dem deutschen Arbeitsmarkt besteht oder ob mit der Einführung einer Bachelor-Master-Studienstruktur eine wissenschaftliche Grundausbildung der Studierenden unwiederbringlich verloren geht ${ }^{8}$. Diesem Stand der Diskussion entspricht auch die ausgesprochene Zurückhaltung bei der Umsetzung auf politischer Ebene ${ }^{9}$. Außerhalb der Rechtswissenschaft muss diese Fokussierung überraschen, enthalten doch die Bologna-Vorgaben etliche Elemente, die über die Etablierung europaweit einheitlicher Abschlüsse deutlich hinausgehen. Evaluation und Akkreditierung, Qualitätsmanagement und Wettbewerb um Studierende zählen beispielsweise zu lehr- und lernbezogenen Tiefenstrukturen des künftigen europäischen Studiensystems, deren Einführung in anderen Fachdisziplinen vielfach in der Umsetzung begriffen bzw. bereits umgesetzt ist ${ }^{10}$. Während dort in der Debatte bereits gefragt wird: »Wie studiert man in Bologna? «11, wird in der juristischen Literatur noch der Verlust des »Einheitsjuristen« beklagt.

\section{Hochschulreformen als Impulsgeber für die Lehre}

Zurzeit gerät allerdings die Lehre an den Universitäten zunehmend in den Blick. Dazu trägt insbesondere die Entwicklung bei, dass die Lehre - ebenso wie die Forschung - zu einer Angelegenheit der Universität als Organisation wird ${ }^{12}$. Der tief greifende Prozess der Hochschulreformen soll unter anderem eine Transformation der Universitäten von lose gekoppelten Systemen in korporative Akteure bewirken, die eine Voraussetzung für strategiefähiges Handeln und Ergebnisverantwortung darstellt, wie es das Modell des New Public Management voraussetzt ${ }^{13}$. Diese Entwicklung wird etwa von der Exzellenzinitiative, aber auch von der europäischen Forschungsförderung gleichermaßen vorausgesetzt und weiter befördert ${ }^{14}$. Dass die zunehmende Adressierung der Universitäten als Organisationen nicht nur im Bereich der Forschung relevant wird, zeigen die Diskussionen um eine Exzellenzinitiative für die Lehre oder eine Erweiterung der bestehenden Exzellenzinitiative um Aspekte der Lehre $^{15}$. Ein Erfolg bei einem entsprechenden wettbewerblichen Verfahren setzt dann voraus, dass Universitäten und ihre Fakultäten Mechanismen und Verfahren entwickeln, die die Lehre thematisieren und fortentwickeln. Insofern wird die individuelle Verantwortlichkeit der Lehrenden an der Hochschule für den Erfolg ihrer Lehrveranstaltungen, die bekanntermaßen vielfach nicht zu einer Priorisierung der Lehre gegenüber der Forschung geführt hat, mit einer institutionellen Verantwortlichkeit verkoppelt.

Ein wesentliches Element des New Public Managements stellt zudem ein Umschwenken von der Input- zur Outputsteuerung dar. Nicht mehr die eingesetzten Ressourcen, sondern - jedenfalls vom Konzept her - die damit erzielten Ergebnisse sollen relevant sein. Daran sind die öffentlichen Einrichtungen und ihre Leistungen zu messen. Dies setzt jedoch voraus, dass Leistungen der Institution überhaupt systematisch erfasst werden. Das führt dazu, dass neben der - mit großen Schwierigkeiten behafteten - Erfassung der Forschung auch die Lehre an den Universitäten zunehmend vermessen wird. Die Installation von Qualitätssicherungssystemen, interne und externe Evaluationen der Lehre sowie die steigende Zahl der Rankings von Fachbereichen und Universitäten verleihen dem Ausdruck ${ }^{16}$. Auch wenn dadurch erzeugter Druck zur Verbesserung der Lehre grundsätzlich zu begrüßen ist, stellt sich die Frage, ob man Leistungen in der Lehre - jedenfalls auf diesem Generalisierungsniveau - sinnvoll messen kann. Die Indikatoren insbesondere der von Zeitungen und Magazinen vorgenommen Rankings und deren Methodik geben jedenfalls durchaus Anlass zur Kritik ${ }^{17}$. Dazu kommt, dass der so entfaltete Konkurrenzdruck nur dann sinnvoll wir- ken kann, wenn Anstrengungen einzelner Fakultäten zur Verbesserung der Lehre sich auch in einer verbesserten Position im Ranking der Fakultäten niederschlagen - und vice versa.

Dieser Prozess wird zudem durch die nahezu flächendeckende Einführung von Gebühren für das Erststudium verstärkt. Entsprechend der beständigen Argumentation der Befürworter solcher Gebühren erzeugen diese den Druck, erwartete Gegenleistungen in der Lehre und der Betreuung auch zu erhalten. Gleichzeitig führt die Frage nach einer angemessenen Verwendung der Studiengebühren dazu, dass Fakultäten und Universitäten die Stärken und Schwächen der universitären Lehre mehr in den Blick nehmen müssen, damit Verbesserungsmöglichkeiten identifiziert und Allokationsentscheidungen getroffen werden können. Diese allgemeine Entwicklung wird für die Rechtswissenschaft noch dadurch zugespitzt, dass sich mit der Bucerius Law School eine zusätzliche Konkurrenz zu den staatlichen Fakultäten im Wettbewerb um prospektive Studierende etabliert hat.

Diese Tendenzen müssen dazu genutzt werden, die Entwicklung einer rechtswissenschaftlichen Fachdidaktik voranzutreiben. Dabei muss aber zunächst das Augenmerk darauf gerichtet werden, an welche Entwicklungslinien eine rechtswissenschaftliche Fachdidaktik anknüpfen kann. Die regelrechte Sprachlosigkeit der Disziplin, wenn es um die rechtswissenschaftliche Lehre geht, verwundert. Das gilt umso mehr, wenn man einen Blick auf die anglo-amerikanischen Systeme wirft, die mit einer reichhaltigen Literatur, bis hin zu eigenen Zeitschriften, aufwarten können, zu der es kein deutsches Pendant gibt ${ }^{18}$. Davon, dass sich etwa die Staatsrechtslehrer- oder Zivilrechtslehrertagung mit zeitgemäßem Rechtsunterricht befasst hat, hat man jedoch noch nichts gehört ${ }^{19}$.

8 Siehe statt vieler etwa Hirte/Mock JuS-Extraheft 12/2005, 3 ff.; KatZenSTEIN DÖV 2006, 709 ff.; SEEWALD Juristenausbildung und Bologna 2007; JeEP DÖV 2007, 411 ff.; SснӧвеL in: Baldus/Finkenauer/Rüfner (Hrsg.) Juristenausbildung in Europa zwischen Tradition und Reform 2008, $331 \mathrm{ff}$

9 Siehe nur den Beschluss der Herbstkonferenz der Justizministerinnen und Justizminister vom November 2008 http://www.justiz.nrw.de/WebPortal/ JM/justizpolitik/jumiko/beschluesse/2008/herbstkonferenz08/I_1.pdf

(Stand 14. März 2009), mit dem der Koordinierungsausschuss beauftragt wurde, über die Einführung von Bachelor/Master-Studiengängen in der Rechtswissenschaft spätestens im Jahr 2011 wieder zu berichten.

10 Vgl. den Bericht der Kommission an den Rat über die Entschließung des Rates vom 23. November 2007 zur Modernisierung der Universitäten im Hinblick auf die Wettbewerbsfähigkeit Europas in einer globalen wissensbasierten Wirtschaft, KOM 2008, 608 endg.

11 Vgl. Huber in: Kehm (Hrsg.) Hochschule im Wandel 2008, 295 ff.

12 KrüCKen/Meier in: Drori/Meyer/Hwang (Hrsg.) Globalization and Organization 2006, $241 \mathrm{ff}$.

13 Vgl. ausführlich zu Konzept und Stand der Hochschulreformen Trute/ Pilniok in: Jansen (Hrsg.) Neue Governance für die Forschung 2009, $21 \mathrm{ff}$

14 KRÜCKen Die Transformation der Universität? Überlegungen zu den Effekten von Exzellenzprogrammen in: Hornbostel/Simon/Heise (Hrsg.) Exzellente Wissenschaft: Das Problem, der Diskurs, das Programm und die Folgen 2008, $73 \mathrm{ff}$.

15 Dazu jüngst der Beschluss der Kultusministerkonferenz vom 6. März 2008 über einen »Wettbewerb Exzellente Lehre« http://www.exzellentelehre.de/pdf/kmk_beschluss_06_03_2008.pdf (Stand 14. März 2009); daneben auch voN MÜNCH »Elite-Universitäten«: Leuchttürme oder Windräder? 2005 S. 63; Hetmeier/Willand in: Statistisches Bundesamt (Hrsg.) Amtliche Hochschulstatistik und Hochschulrankings, Statistik und Wissenschaft, Band 11, 2007, 45 (54). Der Wissenschaftsrat plädiert für die Einrichtung eines nationalen Lehrpreises, vgl. Wissenschaftsrat (Fn. 2) $91 \mathrm{f}$.

16 Vgl. dazu Hornbostel in: Statistisches Bundesamt (Hrsg.) Amtliche Hochschulstatistik und Hochschulrankings, Statistik und Wissenschaft, Band 11, 2007, $6 \mathrm{ff}$.

17 Siehe beispielsweise die Kritik von IPSEN am Ranking des Centrums für Hochschulentwicklung in: Forschung und Lehre 2000, $248 \mathrm{f}$.

18 RöHL (Fn. 1).

19 Ein erhöhtes Engagement der Fachgesellschaften in Fragen der wissenschaftlichen Ausbildung fordert auch der Wissenschaftsrat, vgl. Wissenschaftsrat (Fn. 2) 66. 


\section{Auf dem Weg zu einer rechtswissenschaftlichen Fachdidaktik}

Fasst man den bisherigen Befund zusammen, so zeigt sich ein widersprüchliches Bild. Auf der einen Seite steht eine Entwicklung, die universitäre Lehre zu einer institutionellen Angelegenheit und damit in erster Linie zu einer Sache der Fakultäten zu machen. Auf der anderen Seite findet sich eine hochschuldidaktische Entwicklung, die zwar eine ganze Reihe von zu rezipierenden allgemeinen Überlegungen zum Lehren, Lernen und Prüfen in der Hochschule angestellt hat, aber bisher schon aus strukturellen Gründen kaum zu fachspezifischen Überlegungen vorgedrungen ist. Gleichzeitig haben sich auch diejenigen, die an der Universität Rechtswissenschaft lehren, kaum je mit Fragen einer fachspezifischen Didaktik befasst. Die Zeit ist allerdings reif für die Entwicklung einer rechtswissenschaftlichen Fachdidaktik. Dabei stellen sich verschiedene Aufgaben. Zunächst sind allgemeine Anforderungen an eine rechtswissenschaftliche Fachdidaktik zu skizzieren (1.). Dabei sollte, soweit möglich, ein Anschluss an die hochschuldidaktische Debatte hergestellt werden, insbesondere mit einem Perspektivenwechsel von der Lehrzur Lernorientierung (2.). Daraus ergeben sich verschiedene Handlungsfelder, denen sich die rechtswissenschaftliche Fachdidaktik in einem ersten Zugriff zuwenden könnte (3.).

\section{Anforderungen an eine rechtswissenschaftliche Fachdidaktik}

Bevor einzelne Handlungsfelder einer juristischen Fachdidaktik - sozusagen als Gestaltungsspielraum der Lehrenden - in den Blick genommen werden können, ist zu fragen, was rechtswissenschaftliche Fachdidaktik eigentlich ausmachen soll.

Nach verbreitetem Verständnis in der Rechtswissenschaft wird Didaktik zumeist auf Methodik reduziert. Didaktik liefert danach Hinweise und Methoden zur Gestaltung von Lehrveranstaltungen. Folgt man dieser Überzeugung, handelt es sich bei Didaktik um eine Art Verpackungswissenschaft für das wichtiger erachtete Fachwissen ${ }^{20}$. Wollen Lehrende mit diesem methodenorientierten Verständnis von Didaktik ihre Veranstaltungen »didaktisch" verbessern, setzen die Bemühungen in der Regel bei der Rhetorik des Vortrags oder dem Layout von Folien für den Overhead-Projektor oder die PowerPoint-Präsentation an. Didaktik ist jedoch nicht auf solche Einzelfragen zu reduzieren. Vielmehr gilt es Anschluss an die transdisziplinäre Diskussion und Forschung zur Hochschuldidaktik zu suchen. Unabhängig von den verschiedenen Forschungsansätzen wird hier Didaktik allgemein als die Verknüpfung der lernzielabhängigen Auswahl von Inhalten mit Methodenentscheidungen verstanden $^{21}$. Didaktik ist damit die $»$ Wissenschaft, Lehren auf Lernen $\mathrm{zu}$ beziehen ${ }^{22}$. In der Konsequenz ist gute Lehre »eine Lehre, die studentisches Lernen bewirkt und die zu hoher Qualität dieses Lernens führt « ${ }^{23}$. Im Vordergrund stehen nach diesem Verständnis vor allem die Funktion und das Ziel einer Lehrveranstaltung. Bevor eine Lehrperson sich für eine bestimmte Lehrmethode entscheidet, muss sie sich also etwa darüber Klarheit verschaffen, was sie in der Lehrveranstaltung erreichen will ${ }^{24}$, worin ihre Aufgabe als Lehrperson bestehen soll, welche Rolle den Studierenden in der Veranstaltung zugedacht ist und nach welchen Kriterien der Veranstaltungsablauf geplant werden muss ${ }^{25}$. Didaktik stellt insoweit den Bezugsrahmen für praktische Methodenentscheidungen bereit.

Das didaktische Handeln der Lehrenden wird entscheidend durch die unterrichtete Disziplin bestimmt. In erster Linie definieren die Inhalte den didaktischen Handlungsspielraum der Lehrpersonen; daneben spielen häufig Traditionen und tatsächliche Rahmenbedingungen eine Rolle. Beispielsweise dominieren aufgrund der Studierendenzahlen an den juristischen Fakultäten in Deutschland (Groß-)Vorlesungen ${ }^{26}$ und begleitende
Arbeitsgemeinschaften oder Tutorien als Veranstaltungsformen das Bild. Seminare stellen nur eine punktuelle Form der rechtswissenschaftlichen Lehre dar, die im Wesentlichen auf Studierende im Wahlschwerpunkt, Doktoranden sowie extracurricular Interessierte zugeschnitten ist. Das unterscheidet die rechtswissenschaftliche Ausbildung deutlich von den meisten geistes- und sozialwissenschaftlichen Studiengängen. Der didaktische Handlungsspielraum der Lehrenden wird durch solche disziplinspezifischen Rahmenbedingungen zusätzlich beeinflusst.

Gemessen an den Faktoren, die den didaktischen Handlungsspielraum determinieren, besteht insofern ein Bedarf für eine disziplinspezifische Fachdidaktik. Eine rechtswissenschaftliche Fachdidaktik berücksichtigt deshalb fachtypische inhaltliche Zwänge, wie etwa die normative Ausrichtung der Rechtswissenschaft oder die Fallorientierung im Hinblick auf die Rechtspraxis. Sie beachtet aber auch gewachsene Ausbildungs- und Prüfungsstrukturen (z. B. Unterscheidung zwischen Studium und Referendariat) und orientiert sich am Absolventenprofil, das Ergebnis der Ausbildung sein soll ${ }^{27}$. Schließlich müssen disziplinspezifische Rahmenbedingungen beachtet werden, wie die Anzahl von Studierenden in den Lehrveranstaltungen oder zeitliche Ressourcen von Lehrenden und Studierenden.

\section{Von der Lehr- zur Lernorientierung}

Der rechtswissenschaftlichen Lehre liegt vielfach noch ein relativ simples - objektivistisches - Lernverständnis zugrunde, dass man als Sender-Empfänger-Modell beschreiben kann. Es gibt ein feststehendes Ziel, das Stoffvermittlung heißt, und dann ist es Aufgabe der Lehrenden, daraus handhabbare Pakete zu schneiden und abzusenden, im Sinne einer Fremdsteuerung des Lernprozesses ${ }^{28}$. In dieser Perspektive setzen Verbesserungen in der Lehre an der Übermittlungsleistung an und fragen danach, wie diese Pakete hübscher verpackt werden können. Dabei wird die Subjektivität des Lernprozesses nicht berücksichtigt. Versteht man Lernen dagegen als Wissenskonstruktion ${ }^{29}$, bedeutet dies für die Lehre, dass Studierende nicht lediglich passive Empfänger sind, sondern autonome Lernende, denen durch die Lehre Aneignungsprozesse ermöglicht werden sollen. Hochschuldidaktische Forschungen zeigen, dass Zielorientierung, Selbststeuerung und Eigenaktivität als Merkmale des Lernprozesses an Hochschulen besonders relevant sind ${ }^{30}$. Dies entspricht den Grundannahmen der systemisch-konstruktivistischen Kon-

20 So zu Recht kritisch Flender in: Stelzer-Rothe (Hrsg.) Kompetenzen in der Hochschullehre 2005, 170 (171).

21 Diese häufig gebrauchte Definition geht auf Web LER zurück. Siehe dazu DERs. Das Hochschulwesen 1991, $243 \mathrm{ff}$.

22 Vgl. Wild in: Welbers (Hrsg.) Studienreform mit Bachelor und Master 2001, 29 (30 f).

23 Winteler Lehrqualität = Lernqualität?, Das Hochschulwesen 2002, 42.

24 Insbesondere kann danach differenziert werden, ob es um das Erreichen von Können, also das Automatisieren von Fähigkeiten zu geistigen Fertigkeiten, um das Problemlösen durch Denken, Verstehen und Einsicht, das Behalten und Präsenthalten von Wissen, die Übertragung auf andere Gebiete, also Wissenstransfer geht; ein Überblick über das Spektrum von Lernen findet sich bei SEeL Psychologie des Lernens 2002, 19 m. w. N.

25 Zur Funktions- und Zielorientierung siehe ausführlich Flender (Fn. 20) sowie Pfäffli Lehren an Hochschulen - eine Hochschuldidaktik für den Aufbau von Wissen und Kompetenz 2006, $74 \mathrm{ff}$.

26 Siehe hierzu auch die Glosse von Naucke KJ 1997, $240 \mathrm{ff}$

27 Siehe hierzu beispielsweise $\$ 1$ des Hamburgischen Juristenausbildungsgesetzes vom 11.6.2003, HmbGVBl 2003, 156, zuletzt geändert durch Gesetz vom 20.5. 2005, HmbGVBl 2005, 141 oder $\$ \$ 1$ ff. der Verordnung über die Prüfungsgegenstände der staatlichen Pflichtfachprüfung im Rahmen der ersten Prüfung (PrüfungsgegenständeVO) vom 23. 12. 2003, HmbGVBl 2004, 1.

$28 \mathrm{Zu}$ Objektivismus und Subjektivismus als Lehrparadigmen siehe PFäFFLI (Fn. 25) $22 \mathrm{f}$.

29 Vgl. Seel (Fn. 24) 19

30 PFäffli (Fn. 25) 21 m.w. N. 
zepte für die Erwachsenenbildung ${ }^{31}$, auf denen aufgebaut werden kann. Nimmt man die systemische Perspektive insoweit ernst, dann sind Lernende als psychische Systeme zu betrachten, für die die Lehrenden eine geeignete Lernumwelt zu schaffen haben $^{32}$. Damit wird »das Lehr-Paradigma durch das Lern-Paradigma ersetzt $\aleph^{33}$, und die Lernenden, also die Studierenden, geraten in den Fokus der Analyse und Gestaltung des Lehr-LernProzesses. Dies bedeutet auch, dass die Autonomie der Lernenden als Prinzip und Determinante dieses Prozesses anerkannt wird. Schließlich impliziert ein solches Verständnis eine Abkehr von absoluten Wahrheitsvorstellungen, die der Individualität des Lernprozesses Rechnung trägt ${ }^{34}$.

In der Konsequenz ist eine Auseinandersetzung mit dem eigenen Rollenverständnis vonnöten ${ }^{35}$. Ein gewandeltes Selbstverständnis der Lehrperson vom »Lehrer« zum Lernhelfer bedingt weder einen Verlust an Autorität noch einen Bedeutungsverlust. Die akademische Lehrperson ist für die Lernenden vor allem wegen ihrer hohen Fachkompetenz von großer Wichtigkeit, die im Lehr-Lern-Prozess abhängig von der didaktischen Qualität ihrer Lehre zur Geltung kommt ${ }^{36}$. Allerdings bringt studierendenorientierte Lehre nicht nur die Anerkennung der Zielgrup$\mathrm{pe}^{37}$, sondern führt auch zur Schaffung eines lernförderlichen Klimas an Fakultäten ${ }^{38}$.

Die Fokussierung der Lernenden und die Subjektivität von Lernen führen $\mathrm{zu}$ einem dynamischen Verständnis des LehrLernprozesses. Zwar bleibt die Vorbereitung einer Lehrveranstaltung unerlässlich, der Lernprozess ist aber letztlich nicht planbar. Dies setzt ein hohes Maß an Flexibilität seitens der Lehrenden voraus. Dabei liegt auf der Hand, dass die Orientierung an den individuellen Lernprozessen in einer Großveranstaltung geringer ist, als in einer Lehrveranstaltung mit einer begrenzten und relativ kleinen Anzahl teilnehmender Studierender. Das ändert aber an der Grundlegung nichts, dass die Stoffvermittlung auch in der Großveranstaltung auf lernende Individuen ausgerichtet sein sollte und nicht auf Belehrung ${ }^{39}$.

\section{Handlungsfelder für die rechtswissenschaftliche Fachdidaktik}

Soll der wünschenswerte »shift from teaching to learning $«^{40}$ gelingen, müssen Lehrende zunächst ihre didaktischen Handlungsoptionen ausloten. Für die Verbesserung der Wissenspräsentation und der Wissensvermittlung bei gleichzeitiger Unterstützung der Wissensaneignung bieten sich verschiedene Handlungsfelder. Die folgenden Anregungen benennen mögliche Bausteine einer rechtswissenschaftlichen Fachdidaktik und stellen gleichsam eine Forschungsagenda dar. Sie nehmen verschiedene Punkte auf, die bereits in der Rechtswissenschaft selbst ${ }^{41}$, in der allgemeinen Hochschuldidaktik oder in der anglo-amerikanischen Debatte um legal education thematisiert werden. Die Elemente sind nicht abschließend, sondern als dynamische und erweiterungsfähige Anregungen zu verstehen.

\section{a) Lernorientierte Arbeitsformen in Lehrveranstaltungen}

Großvorlesungen werden sich in absehbarer Zeit aus Kapazitätsgründen nicht abschaffen lassen - obgleich sie ein lernorientiertes Lehren erschweren. Aber auch dort, wo letzteres möglich ist, sind Juristen bekanntermaßen konservativ. Dies spiegelt sich beispielsweise in der Dominanz des gelenkten Unterrichtsgesprächs in Arbeitsgemeinschaften und Tutorien, das eine Reihe von Nachteilen für die Aktivierung aller Studierenden mit sich bringt. Es müsste daher durch andere Methoden ergänzt werden ${ }^{42}$. Hierbei könnten Anregungen der Hochschuldidaktik aufgenommen ${ }^{43}$ und auf die Besonderheiten der rechtswissenschaftlichen Veranstaltungen, etwa die Fallorientierung in den Arbeitsgemeinschaften, zugeschnitten werden. Als positives Beispiel für den erfolgreichen Einsatz von lernorientierten Arbeitsformen sei die Veranstaltung »Einführung in das rechtswissen- schaftliche Arbeiten « an der Fakultät für Rechtswissenschaft der Universität Hamburg genannt. Hier werden in jeder Unterrichtseinheit Einzel- und Gruppenarbeiten sowie entsprechenden Präsentationen vor der jeweiligen Kursgruppe eingesetzt. Von diesen Erfahrungen könnten kleinere Lehrveranstaltungen in den Wahlschwerpunkten sowie Arbeitsgemeinschaften und Tutorien profitieren.

\section{b) Verknüpfung von Lehrveranstaltungen}

Nach dem Verständnis von Lernen als Wissenskonstruktion setzt das Lernen neuer Inhalte immer am erworbenen Vorwissen und an Vorerfahrungen an. Dabei erleichtern Anknüpfungspunkte an vorhandenes Wissen ${ }^{44}$ und Wiederholungsmöglichkeiten den Lernprozess ${ }^{45}$. In diesem Sinne ist es im Rahmen der Entwicklung einer rechtswissenschaftlichen Didaktik wünschenswert, die Verknüpfung verschiedener Lehrveranstaltungen zu thematisieren. Hier erscheint eine Abstimmung der Lehrkonzepte im Hinblick auf das Curriculum sowohl im Studienverlauf, d.h. Lehrveranstaltungen in vorangegangenen wie nachfolgenden Semestern wie auch mit parallel stattfindenden Lehrveranstaltungen von großer Bedeutung. Dabei können die jeweiligen Lernbedingungen der verschiedenen Veranstaltungsarten stärker als bisher zur Definition unterschiedlicher Lernziele im Sinne einer Aufgabenteilung zwischen verschiedenen Veranstaltungen genutzt werden. So ist insbesondere an die Verschränkung von Vorlesungen und den an nahezu allen Fakultäten eingerichteten vertiefenden Arbeitsgemeinschaften und Tutorien zu denken.

\section{c) Visualisierung}

Zudem kann mit dem Thema Visualisierung an den »derzeit einzige[n] aktive[n] Zugang zur juristischen Didaktik « ${ }^{46}$ angeknüpft werden. Dabei mag die weit verbreitete These zu kurz greifen, dass Menschen regelmäßig 20\% der Inhalte aufnehmen, die sie nur hören, 30\% dessen behalten, was sie sehen, während sie, gleichsam in der Summe, 50\% dessen rezipieren, was sie sowohl hören als auch sehen, weil sie individuelle Wahrnehmungsfaktoren nicht hinreichend berücksichtigt ${ }^{47}$. Allerdings lassen sich wissenschaftlich kaum Zweifel daran begründen, dass es nicht nur motivations-, sondern auch lernfördernd ist, wenn bei der Vermittlung von Inhalten verschiedene Sinne angesprochen werden ${ }^{48}$.

$31 \mathrm{Zu}$ dieser Perspektive Siebert Didaktisches Handeln in der Erwachsenenbildung 5. Aufl. (2006) 259

32 Edelmann Lernpsychologie, 6. Aufl. (2000) 287.

33 Pörksen in: Laske (Hrsg.) Personalentwicklung und universitärer Wandel: Programm - Aufgaben - Gestaltung, 2004, 97 (102).

34 Zum Ganzen Pörksen (Fn. 33) $100 \mathrm{ff}$.

35 Eingehend Wörner Lehren an der Hochschule 2006, $119 \mathrm{ff}$.

36 Siebert (Fn. 31) 260 sowie Pfäffli (Fn. 25) $141 \mathrm{f}$.

37 Wörner (Fn. 35) 130

38 Hierzu Winteler Lehrqualität = Lernqualität?, Das Hochschulwesen 2002, 42 (45).

39 Siebert Didaktisches Handeln in der Erwachsenenbildung 5. Aufl. 2006, $27 \mathrm{ff}$. sowie 261.

40 Wildt (Fn. 22) 29 (37).

41 Siehe etwa Bork JuS 1999, $413 \mathrm{ff}$.

42 Dabei ist zu berücksichtigen, dass das so genannte sokratische Gespräch als didaktisches Modell durchaus voraussetzungsvoller ist als die vorfindlichen Unterrichtsgespräche, vgl. dazu eingehend Siebert (Fn.31) 171 ff. m. w. N. sowie Pörksen (Fn. 33) 109 ff.

$43 \mathrm{Zu}$ eigenverantwortlichem und aktivem Lernen eingehend PFÄFfLI (Fn. 25) S. $90 \mathrm{ff}$.

44 Zielgruppenorientierung kann in diesem Zusammenhang dahingehend verstanden werden, dass nicht allein darauf abgestellt wird, was die Studierenden wissen müssten, sondern darauf, was sie - erfahrungsgemäß tatsächlich wissen, wenn sie eine bestimmte Lehrveranstaltung besuchen.

45 Dummann et al., Einsteigerhandbuch Hochschullehre 2007, 26 ff.

46 RöHl (Fn. 1).

47 Vgl. Klimsa in: Issing/Klimsa (Hrsg.) Information und Lernen mit Multimedia und Internet 3. Aufl. 2002, 5 (9, 13 m. w. N.).

48 Dummann et al. (Fn. 45) $126 \mathrm{ff}$. 
Voraussetzung dafür ist freilich der wohl überlegte und -dosierte Einsatz von Visualierungsmethoden und Medien ${ }^{49}$. Röhl und Ulbrich haben sich in ihrem Buch »Recht anschaulich« gleichermaßen von der theoretischen wie der praktischen Seite der Visualisierung im Rechtsunterricht in äußerst gewinnbringender Weise genähert ${ }^{50}$. Dabei wird deutlich, dass die Visualisierung von Rechtsinhalten für die »bilderscheue Rechtswissenschaft" eine Herausforderung ist, der sie sich stellen muss. Gerade weil die Visualisierung für Ausbildungszwecke nicht voraussetzungslos ist, würde eine breite Befassung mit dem Thema in einer rechtswissenschaftlichen Fachdidaktik es ermöglichen, best practiseBeispiele - wie Röhl und Ulbrich es für das Zivilrecht tun - zur Verfügung zu stellen, aber auch einen Austausch über mit der Visualisierung verbundene Probleme zu initiieren.

Selbstverständlich spielen die räumlichen und technischen $\mathrm{Ge}$ gebenheiten bei der Auswahl der Visualisierungsmethode eine entscheidende Rolle und schränken diese zugleich ein. Daneben sind allerdings weitere Faktoren bei der Methodenauswahl zu berücksichtigen. So sind Vor- und Nachteile der verschiedenen Möglichkeiten gegeneinander abzuwägen, zum Beispiel der Vorbereitungsaufwand, die Aufmerksamkeit, die eine Visualisierungsmethode während der Veranstaltung erfordert und schließlich die Flexibilität im Hinblick auf den Lernprozess zu berücksichtigen. Visualisierungsmethoden mit statischen Inhalten wie PowerPoint-Präsentationen oder vorgefertigte OverheadFolien ohne Ergänzungsmöglichkeiten eignen sich beispielsweise kaum zur Visualisierung der gemeinsamen Fallbearbeitung in Arbeitsgemeinschaften oder Tutorien, weil sie keine Möglichkeit bieten, den gemeinsamen Arbeitsprozess abzubilden, in dem durchaus verschiedene Lösungswege eingeschlagen werden können.

\section{d) E-Learning}

Auch in der juristischen Lehre ist über die Einsatzmöglichkeiten virtueller Lernplattformen nachzudenken ${ }^{51}$. Dabei scheint es derzeit schwer vorstellbar und nicht wünschenswert, Präsenzveranstaltungen durch E-Learning-Angebote zu ersetzen. Allerdings kann eine Mischform aus Präsenzunterricht und Einsatz von E-Learning-Angeboten, also so genanntes Blended-Learning durchaus sinnvoll sein. Dabei lassen sich die Vorteile beider Lehrformen nutzbar machen ${ }^{52}$. Der Präsenzunterricht bietet die persönliche Ansprechbarkeit und den direkten Kontakt zur Lehrperson, dagegen ermöglicht das E-Learning-Angebot den Studierenden eine große Unabhängigkeit ${ }^{53}$. Es soll nicht verschwiegen werden, dass die erstmalige Einrichtung von Lernplattformen in einem ersten Schritt durchaus zeit- und arbeitsaufwändig ist ${ }^{54}$. Allerdings stehen für die Entwicklung und den Einsatz von E-Learning im Hochschulunterricht inzwischen vielerorts entsprechende Infrastrukturen sowie personelle und finanzielle Mittel zur Verfügung. Auch ermöglichen es die Programme, verschiedene Funktionalitäten auszuwählen und die Lernplattform konkret an den Bedürfnissen ausgerichtet für eine Lehrveranstaltung zu gestalten. Zudem können zum Beispiel durch die Bildung von Arbeitsgruppen oder Tandems gegenseitige Lernkontrollen oder Selbstkontrollen durch die Studierenden durchgeführt werden, was anderenorts Korrekturaufwand vermindern kann.

\section{e) »Clinical Legal Education"}

$\mathrm{Zu}$ den festen Elementen der rechtswissenschaftlichen Ausbildung in den Vereinigten Staaten gehört seit mehreren Jahren die Clinical Legal Education. Darunter versteht man eine »juristische Ausbildungsmethode, die Studenten Situationen aussetzt, in denen sie zuvor in der Lehrveranstaltung erarbeitete rechtliche Theorien, Prinzipien und Lehrmeinungen anwenden $\aleph^{55}$. Dazu gehören zunächst die hier bereits bekannten moot courts, die Gerichtsverhandlungen simulieren. Insofern gilt es für die rechtswissenschaftliche Fachdidaktik, Ansatzpunkte zu ent- wickeln, Simulationsübungen auch in anderen Formen und zu anderen Problemen als der gerichtlichen Auseinandersetzung zu gestalten. Dabei können allgemeine hochschuldidaktische Überlegungen zum Einsatz von Planspielen in der universitären Lehre fruchtbar gemacht werden ${ }^{56}$.

Darüber hinaus zählen zu der Clinical Legal Education in den USA neben Praktika insbesondere die live client clinic $^{57}$. Dabei übernehmen Studierende unter intensiver Betreuung der Lehrenden die Rechtsberatung »realer Menschen mit realen Problemen «. Schon in der Bezeichnung an die medizinische Ausbildung angelehnt, wird dies als zentrales Element zur Entwicklung professioneller Kompetenzen gesehen ${ }^{58}$. Auch wenn die Rahmenbedingungen in Deutschland angesichts der Zweiteilung der Ausbildung in Studium und Referendariat und einer unabhängig von wirtschaftlichen Verhältnissen zugängliche Rechtsberatung anders sind, ließe sich diese Veranstaltungsform auch in die rechtswissenschaftliche Ausbildung einbinden. Derartige Ansätze gab es schon im Rahmen der einstufigen Juristenausbildung mit Projekten zur Beratung von Umweltverbänden ${ }^{59}$. Ein aktuelles Modell lässt sich an der Universität Gießen finden, wo die Refugee Law Clinic Theorie und Praxis des Migrationsund Flüchtlingsrechts miteinander verbindet ${ }^{60}$. So lässt sich ein Lebensweltbezug der Ausbildungsinhalte herstellen ${ }^{61}$, der die Sinnhaftigkeit des Lehrangebots veranschaulicht und mindestens motivationsfördernd wirkt ${ }^{62}$.

\section{f) Methodenorientierte Lehrveranstaltungen}

Nimmt man den konstruktivistischen Ansatz ernst, wird man zugestehen müssen, dass selbstbestimmtes und -gesteuertes Lernen ${ }^{63}$ erst durch die Beherrschung disziplintypischer Arbeitstechniken und Arbeitsmethoden ermöglicht wird. Gerade in der Rechtswissenschaft kommt es für die Studierenden nicht nur darauf an, Wissen zu generieren, sondern vor allem auch dieses Wissen zu operationalisieren. Oftmals bereitet diese Operationalisierung bis zur Staatsprüfung Probleme. Vielen Studierenden

49 Weidenmann in: Issing/Klimsa (Fn. 47) 52 ff. m. w. N.

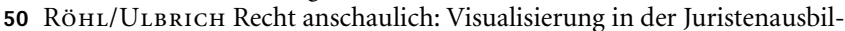
dung 2007; vgl. zur Bedeutung von Visualisierung in juristischen Vorlesungen auch schon Bork (Fn. 41) 415.

51 Überblick über die Situation an deutschen Hochschulen bei KLEInManN in: Merkt et al. (Hrsg.) Studieren neu erfinden, Hochschule neu denken 2008, $149 \mathrm{ff}$.

52 Zur herausragenden Bedeutung der persönlichen Beziehung zwischen Studierenden und Lehrenden in diesem Zusammenhang siehe KaTZLINGER in: Merkt et al. (Fn. 52) $191 \mathrm{ff}$.

$53 \mathrm{Zu}$ den Vorteilen von E-Learning eingehend Schulmeister Gibt es eine Net-Generation, abrufbar unter http://www.zhw.uni-hamburg.de/uploads/schulmeister-net-generation_v2.pdf (Stand 14. März 2009), $83 \mathrm{ff}$. m. w. N.

$54 \mathrm{Zu}$ den Vor- und Nachteilen der Einsatzes von E-Learning siehe auch Dummann et al. (Fn. 45) $109 \mathrm{f}$.

55 Siehe dazu den instruktiven Überblick bei BücKer/WoodrufF JZ 2008, $1068 \mathrm{ff}$

56 Vgl. Dummann et al. (Fn. 45) 89 ff. zu Planspielen.

57 BÜCKER/WOOdruff (Fn. 55) $1072 \mathrm{f}$.

58 Zum Professionsbegriff vgl. Stichwen Ius Commune 19, 1992, $279 \mathrm{ff}$.

59 So gab es in Bremen Projekte zur Rechtsberatung von Mieter- und Umweltinitiativen durch Studieren, siehe Winter Rechtsberatung als Projektbestandteil in: Francke/Hart/Lautmann/Thoss (Hrsg.) Einstufige Juristenausbildung in Bremen 1982, $80 \mathrm{ff}$.

$60 \mathrm{http} / /$ www.recht.uni-giessen.de/wps/fb01/proj/det/Gross/32/(Stand 14. März 2009).

61 Siehe auch IвEN in: Fritsch/Maraun (Hrsg.) Über ein anderes Bild von Lehre 1998, $47 \mathrm{ff}$.

62 Siehe auch das Projekt "Studentische Rechtsberatung " an der FHVR, http://www.fhvr-berlin.de/fhvr/index.php?id=423\&color=basis\&size $=100$ (Stand 14. März 2009), das zwar an einer Fachhochschule entwickelt worden ist, jedoch auf die universitäre Ausbildung übertragbar erscheint.

63 Zur Bedeutung metakognitiven Lernens im Studium Seel (Fn. 24) 233 ff., insbesondere zur Förderung der Studierfähigkeit, 237 f. je m. w. N. 
fehlen ganz elementare Grundkenntnisse (rechts-)wissenschaftlichen Arbeitens. In den - soweit ersichtlich an allen Fakultäten angebotenen - vorlesungsbegleitenden Arbeitsgemeinschaften oder Tutorien, in denen Fallbearbeitung eingeübt werden soll, spielt die Vermittlung materiellen Wissens eine so große Rolle, dass eine Konzentration auf die Arbeitstechniken nicht möglich ist. Methodenorientierte Lehrveranstaltungen zu Beginn des Studiums können diesem Defizit wirkungsvoll begegnen. Sie können Sorge dafür tragen, die Studierenden in die Lage zu versetzen, in Lehrveranstaltungen oder durch Lektüre angeeignetes materielles Wissen auch umsetzen zu können. Erst die Befähigung zur Umsetzung ermöglicht insoweit den Lernprozess.

Verschiedene deutsche Fakultäten haben diesen Gedanken aufgegriffen und bieten propädeutische Veranstaltungen zum rechtswissenschaftlichen Arbeiten für Erstsemester an. In Anlehnung an die in der US-amerikanischen Hochschullandschaft üblichen Veranstaltungen zu »Legal Research and Writing« wird den Studierenden Gelegenheit gegeben, die Grundlagen rechtswissenschaftliches Arbeiten kennen zu lernen und einzuüben ${ }^{64}$.

\section{g) Lernkontrollen}

Schließlich muss über die Form der Rückmeldungen an Studierende über ihren Leistungsstand nachgedacht werden. Der Wissenschaftsrat hat jüngst darauf hingewiesen, dass Studierende in keinem Fach die Praxis des Feedbacks so schlecht bewerten wie in der Rechtswissenschaft ${ }^{65}$. Dafür bilden auch die vielfachen Anträge auf eine Nachkorrektur von Klausuren ein deutliches Indiz. Das sollte Anlass zu einer Weiterentwicklung der herkömmlichen Methoden geben ${ }^{66}$. Insbesondere darf bezweifelt werden, ob Leistungsnachweise im rechtswissenschaftlichen Studium überhaupt als Lernkontrollen ausgelegt sind und die Studierenden zuverlässig über ihren Lernstand informieren. Der Bezug zwischen Unterricht und Prüfung ist in der Rechtswissenschaft traditionell besonders locker; letztlich ist der Konnex vielfach nur in der Eingrenzung auf ein Themengebiet in einer Klausur zu suchen. Dazu kommt die an vielen Universitäten gepflegte Praxis, dass die Korrektur der Leistungskontrollen extern vergeben wird und deshalb außerhalb der Lehrveranstaltungen erfolgt ${ }^{67}$. Diese auf die hohen Studierendenzahlen zurückzuführende Entwicklung führt $\mathrm{zu}$ erheblichen Instruktions- und Kontrollproblemen und lockert die Bindung an eine Lehrveranstaltung und deren Inhalte stark $^{68}$. Eine Möglichkeit zur Veränderung ist etwa in der Einführung von Lernkontrollen unterhalb der Leistungskontrollen zu sehen. Das bietet sich insbesondere für die Arbeitsgemeinschaften an, wo Studierenden in »Hausaufgaben « Gelegenheit gegeben werden kann, die Inhalte des Präsenzunterrichts zu vertiefen und schriftlich niederzulegen. Damit ist freilich eine deutliche Erhöhung der Arbeitslast für DozentInnen verbunden. Ein weiterer Ansatzpunkt zur Verbesserung könnte hier zudem das Lernen von anderen Hochschulsystemen sein. So hat etwa das Fachdidaktikzentrum für die rechtswissenschaftliche Ausbildung in Großbritannien umfangreiche Arbeiten zur Frage vorgelegt, wie Studierende didaktisch sinnvoll Feedback für ihre Leistungen bekommen können ${ }^{69}$. Auch wenn man unterschiedliche Hochschul-, Ausbildungs- und Rechtssysteme durchaus in Rechnung stellen muss, können diese Arbeiten vielfältige Anregungen für die rechtswissenschaftliche Fachdidaktik geben.

\section{Infrastruktur für eine rechtswissenschaftliche Fachdidaktik}

Wie bereits festgestellt, ist gute Lehre nicht nur von den Beteiligten abhängig, sondern auch von förderlichen Rahmenbedingungen. Die Entwicklung einer rechtswissenschaftlichen Fachdidaktik bedarf insoweit institutioneller Weichenstellungen auf verschiedenen Ebenen.

\section{Fakultäts- und Universitätsebene}

Auf Fakultäts- und Universitätsebene gerät zunächst die Anreizsteuerung in den Blick. Verschiedentlich bieten sich Ansatzpunkte, gute Lehre messbar zu honorieren und dadurch die Entwicklung der rechtswissenschaftlichen Fachdidaktik zu befördern.

Im Zusammenhang der internen Hochschulsteuerung wird etwa seit langem eine lehr-leistungsbezogene Mittelvergabe diskutiert ${ }^{70}$. Sicherlich birgt die Schaffung eines wettbewerblichen Anreizrahmens, der Aufgabenerfüllung, Leistung und Innovationsfähigkeit finanziell belohnt, gewisse Risiken und Probleme ${ }^{71}$. Allein die Erfassung und Vergleichbarkeit von Lehrleistungen dürfte vielfach schwer fallen. Lehr-leistungsbezogene Mittelvergabe sollte sich deshalb daraufbeschränken, positive Entwicklungen zu fördern; in keinem Fall darf sie finanziell sanktionieren ${ }^{72}$.

Ein weiterer Ansatzpunkt für die Anreizsteuerung kann die Reputation für Lehrleistungen sein. Bislang stellen ausschließlich Forschungsarbeiten die Währung wissenschaftlicher Reputation dar. So werden zukünftige HochschullehrerInnen an den juristischen Fakultäten bis heute im Wesentlichen aufgrund der Qualität und Quantität ihrer Forschungsleistungen berufen. Als Gegengewicht zum Wissenschaftssystem muss deshalb mehr Reputation für Lehrleistungen erzeugt werden ${ }^{73}$. Als Instrument dafür eignet sich nicht nur die universitäts- oder fakultätsöffentliche Auszeichnung für gute Lehre. Auch muss im Rahmen der akademischen Stellenbesetzung eine didaktische Qualifizierung eine deutlich größere Rolle als bisher spielen. Dies gilt insbesondere für die Berufung zukünftiger HochschullehrerInnen. Nach wie vor finden entsprechende normative Zielvorgaben der Hochschulgesetze ${ }^{74}$ in den Auswahlverfahren kaum Anwendung. Zwar

64 So zum Beispiel an der Universität Frankfurt (»Einführung in die juristische Arbeitstechnik «), an der Universität Hamburg (»Einführung in das rechtswissenschaftliche Arbeiten«), an der Universität Leipzig (»Einführung in die juristische Argumentation und Methodik mit integriertem Moot Court«) sowie an der Universität Passau (»Einführung in das wissenschaftliche Arbeiten «). Allerdings handelt es sich jeweils um verschieden Veranstaltungsformen (Vorlesung, Kleingruppen oder Mischformen) mit unterschiedlichen didaktischen Konzepten.

65 Wissenschaftsrat (Fn. 2) 32.

66 Das gilt umso mehr angesichts empirischer Untersuchungen, dass Erfolgserwartung und Erfolgserlebnisse zentrale Gesichtspunkte von Leistungsmotivation sind. Dagegen beeinträchtigt die Furcht vor Misserfolg nicht nur die Leistungsmotivation sondern auch die Lernfähigkeit nachhaltig, vgl. Edelmann Lernpsychologie 6. Aufl. 2000, 45. Dies spricht dafür, geschützte Lernräume zu schaffen, in denen die Rückmeldung zum Lernfortschritt nicht gleichzeitig zu einer Leistungsbewertung führt.

67 Es sind jedoch auch auf die Verwendung von Studiengebühren zurückzuführende gegenläufige Tendenzen erkennbar. So setzt die Universität Freiburg Lehrassistenten ein, die Klausuren in den Fortgeschrittenenübungen korrigieren. Zudem sind etwa an den Universitäten Hamburg, Hannover und München sog. »Klausurenkliniken« eingerichtet worden, die als Anlaufstellen für Studierende dienen, die ein erweitertes Feedback zu ihren Klausuren erhalten möchten.

68 Zur Relevanz der Konsistenz von Lehrperson, Lernprozess und Lernkontrolle siehe Pfäffli (Fn. 25) $153 \mathrm{f}$.

69 Vgl. die Materialien unter http://www.ukcle.ac.uk/resources/assessment/ index.html (Stand 14. März 2009).

70 Siehe ausführlich JAEger HIS 12/2005, 1 (5 ff.).

71 Siehe dazu Schimank Forschung \& Lehre 1998, 72 ff.

72 So hat die Fakultät für Rechtswissenschaft der Universität Hamburg jüngst diesen Gedanken aufgegriffen und zwischen den Schwerpunktbereichen einen Wettbewerb um Konzepte zur Verbesserung der Schwerpunktausbildung veranstaltet, in dem die besten Konzepte mit $€ 20.000$ prämiert worden sind.

73 Zum Verhältnis von Forschung und Lehre siehe auch RumpF in: Fritsch/ Maraun (Hrsg.) Über ein anderes Bild von Lehre 1998, 9 ff. sowie SCHIMANK Haben Professoren weniger Zeit für die Forschung? in: dvs-informationen 1996, $47 \mathrm{ff}$.

74 Vgl. zum Beispiel \$47 I Nr. 2 des Landeshochschulgesetzes Baden-Württemberg: Die pädagogische Eignung für die Lehre an der Hochschule ist eine Einstellungsvoraussetzung für Professorinnen und Professoren. Die Regelung findet sich nahezu wortgleich in allen Landeshochschulgesetzen. 
ist auch hier eine Operationalisierung, die auch verfahrensrechtlichen Anforderungen standhält, nicht ganz leicht. Der Wissenschaftsrat schlägt unter anderem die Einführung eines Lehrportfolios in den Berufungsverfahren vor ${ }^{75}$. Ein Lehrportfolio ist eine Darstellung der bisher erbrachten Lehrleistungen und der dazu angestellten didaktischen Überlegungen. Daneben sollten Lehrproben zu vorgegebenen Themen den KandidatInnen die Möglichkeit geben, ihr Können etwa hinsichtlich der Stoffauswahl oder der Vermittlungsmethoden unter Beweis zu stellen.

Ein besonderes Augenmerk sollte überdies der Ausbildung des wissenschaftlichen Nachwuchses gelten. Die wissenschaftlichen MitarbeiterInnen bilden vielfach das Rückgrat der rechtswissenschaftlichen Ausbildung, indem sie Arbeitsgemeinschaften und Tutorien leiten, die nahezu alle Fakultäten - nicht zuletzt mit Hilfe der Studiengebühren - flächendeckend eingerichtet haben. Die wissenschaftlichen MitarbeiterInnen führen diese Lehrveranstaltungen in aller Regel ohne jede didaktische Qualifizierung und Anleitung durch. Gründe hierfür liegen im mangelnden Interesse der Fakultäten an Qualifizierungsmaßnahmen, der verbreitet hohen Arbeitsbelastung der wissenschaftlichen MitarbeiterInnen sowie der fehlende rechtswissenschaftliche Fachdidaktik. Hier Abhilfe zu schaffen stellt eine vordringliche Aufgabe und Herausforderung für die rechtswissenschaftlichen Fakultäten in Deutschland dar ${ }^{76}$.

Schließlich ist der Tatsache Rechnung zu tragen, dass die Hochschullehre und das Vermitteln von Inhalten an Studierende für die Lehrenden selbst mit einem ständigen Lernprozess einhergeht ${ }^{77}$. Daher ist es wünschenswert und erforderlich, an den Fakultäten insofern eine Lernumwelt zu schaffen, in der - idealerweise in fehlerfreundlicher Atmosphäre - der Austausch über didaktische Fragen und Probleme der Lehre ermöglicht und gefördert wird.

\section{2. Übergreifende Ebene}

Um Konsequenzen aus der fehlenden fachspezifischen Ausdifferenzierung der Didaktikzentren an den Universitäten zu ziehen, hat der Wissenschaftsrat die Einrichtung von Fachdidaktikzentren vorgeschlagen, die jeweils für ein Fach oder eine Fächergruppe bundesweit zentral geschaffen werden ${ }^{78}$. Die Idee der Fachdidaktikzentren orientiert sich am englischen Modell ${ }^{79}$. Dort sind 24 sog. Subject Centres eingerichtet worden, die jeweils disziplinäre Lehrfragen bearbeiten. So ist an der Universität Warwick das UK Centre for Legal Education eingerichtet worden ${ }^{80}$. Der derzeitige Direktor des Zentrums, Julian Webb, hat sogar eine Professur an der Universität Warwick inne, die Legal Education gewidmet ist. $\mathrm{Zu}$ den Aufgaben zählen etwa die Erarbeitung disziplinärer Lehrstandards, die Entwicklung von Lehr- und Studienmaterialien, die Durchführung von Modellprojekten zur Fortentwicklung der Lehre, die Herausgabe von Fachzeitschriften und die Durchführung von Fortbildungsveranstaltungen. Solche Fachdidaktikzentren sollen in einem wettbewerblichen Verfahren, das bisherige Leistungen einer Fakultät in Forschung und Lehre als Bewertungskriterien heranzieht, für einen befristeten Zeitraum vergeben werden. Damit würden wesentliche infrastrukturelle Voraussetzungen für den Ausbau der rechtswissenschaftlichen Fachdidaktik geschaffen. Mit der Herausgabe einer Fachzeitschrift würde insbesondere eine Lücke geschlossen, die im Vergleich zu den Vereinigten Staaten und Großbritannien besteht. In den USA zählt das Journal of Legal Education zum traditionellen Kanon der juristischen Zeitschriften, in England erscheint die Zeitschrift The Law Teacher ${ }^{81}$. Die permanente Thematisierung aktueller fachdidaktischer Fragen sowie die Schaffung einer zentralen Anlaufstelle dürften den Stellenwert der rechtswissenschaftlichen Fachdidaktik deutlich erhöhen.

\section{Zusammenfassung}

Verschiedene Faktoren haben das Entstehen einer rechtswissenschaftlichen Fachdidaktik bisher behindert, obwohl ein Bedarf seit langem besteht. Im Fahrwasser der Hochschulreformen erscheint es nun denkbar, die Entwicklung einer rechtswissenschaftlichen Fachdidaktik voranzutreiben. Dabei gilt es zunächst, Anschluss an die allgemeine hochschuldidaktische Debatte zu suchen und disziplinspezifische Handlungsfelder zu identifizieren. Eine dauerhafte Etablierung rechtswissenschaftlicher Fachdidaktik kann jedoch nur gelingen, wenn insbesondere auf Universitäts- und Fakultätsebene eine förderliche Infrastruktur geschaffen wird.

75 Wissenschaftsrat (Fn. 2) 43 f., 68. Das ist freilich nicht ganz neu, vgl dazu den bei RöHL Hier war die Rechtsdidaktik, http://rsozblog.de/ ?cat=90 (Stand 14. März 2009), wiedergegebenen Text von WindeL zu seinen Vorstellungen über die akademische Lehre anlässlich eines Berufungsverfahrens.

$76 \mathrm{Zu}$ den Anforderungen an die Gestaltung hochschuldidaktischer Weiterbildung siehe Reiber Wissen - Können - Handeln, Ein Kompetenzmodell für lernorientiertes Lehren, Tübinger Beiträge zur Hochschuldidaktik 2006, 9 ff., abrufbar unter http://w210.ub.uni-tuebingen.de/dbt/ volltexte/2006/2296/pdf/TBHD_2-1_(2006)_Reiber.pdf (Stand 14. März 2009).

77 Shulman in: Wilson (Hrsg.) The Wisdom of Practice: Essays on Teaching, Learning, and Learning to teach 2004, $189 \mathrm{ff}$., DERs. Teaching alone, learning together: needed agendas for the new reforms, a. a. O. 311 ff., WÖrNER (Fn. 35) 129.

78 Wissenschaftsrat (Fn. 2) 70 ff. Darauf zielt jetzt auch die Förderinitiative »Bologna - Zukunft der Lehre« der Volkswagenstiftung und der Stiftung Mercator, vgl. http://www.volkswagenstiftung.de/foerderung/ strukturen-und-personen/hochschule-der-zukunft.html (Stand 14. März 2009). Innerhalb dieses Programms können sich Universitäten unter anderem um Mittel für den Aufbau von Zentren für Lehr- und Lernforschung sowie Curriculumforschung bewerben.

79 Wissenschaftsrat (Fn. 2) 72.

80 Informationen darüber sind unter www.ukcle.ac.uk abrufbar (Stand 14. März 2009).

81 RöHL (Fn. 1). 